\title{
Characterization of entanglement transformation via group representation theory
}

\author{
Li-Xiang Cen ${ }^{1,2}$, Xin-Qi $\mathbf{L i}^{1,2}$ and Yi Jing Yan ${ }^{1}$ \\ ${ }^{1}$ Department of Chemistry, Hong Kong University of Science and Technology, Kowloon, \\ Hong Kong \\ 2 NLSM, Institute of Semiconductors, Chinese Academy of Sciences, PO Box 912, \\ Beijing 100083, People's Republic of China \\ E-mail: cenlx@chsg4.ust.hk and yyan@ust.hk
}

Received 18 June 2003

Published 25 November 2003

Online at stacks.iop.org/JPhysA/36/12267

\begin{abstract}
Entanglement transformation of composite quantum systems is investigated in the context of group representation theory. Representation of the direct product group $S L(2, C) \otimes S L(2, C)$, composed of local operators acting on the binary composite system, is realized in the four-dimensional complex space in terms of a set of novel bases that are pseudo-orthonormalized. The two-to-one homomorphism is then established for the group $S L(2, C) \otimes S L(2, C)$ onto the $S O(4, C)$. It is shown that the resulting representation theory leads to the complete characterization for the entanglement transformation of the binary composite system.
\end{abstract}

PACS number: $03.65 . \mathrm{Ud}$

Nonlocal correlation or entanglement between spatially separated quantum systems is one of the most striking features of quantum mechanics. In recent years, extensive attention has been paid to this topic due to its great importance in quantum information processing [1-3]. One basic approach to this subject, first exploited by Bennett and co-workers $[4,5]$, is to employ entanglement transformation for quantum states under actions of local quantum operations and classical communication (LOCC). Such studies have contributed to characterizing what is possible locally and what is impossible, promising in principle a criterion not only for distinguishing entangled (nonseparable) states from separable states but also for classifying entanglement in different categories. Entanglement transformation also relates to the measures of entanglement. For instance, the asymptotic measures such as distillable entanglement and entanglement cost owe their very existence to this body of knowledge [6, 7]. 
Despite being very fruitful for pure entangled states [8-10], the entanglement transformation has so far provided only fragments of knowledge on mixed entangled states [11-13], even for the simplest binary composite system. Consider a quantum system described by a density matrix $\rho$ in the Hilbert space $H_{A} \otimes H_{B}$. State transformation under the actions of LOCC can be illustrated as [14]

$$
\rho \rightarrow \rho^{\prime}=\frac{\sum_{i, j} A_{i} \otimes B_{j} \rho A_{i}^{\dagger} \otimes B_{j}^{\dagger}}{\sum_{i, j} \operatorname{tr}\left(A_{i}^{\dagger} A_{i} \otimes B_{j}^{\dagger} B_{j} \rho\right)}
$$

where $A_{i}$ and $B_{j}$ are local operators acting on the two isolated subsystems, obeying the conditions $\sum_{i} A_{i}^{\dagger} A_{i} \leqslant I_{2}$ and $\sum_{j} B_{j}^{\dagger} B_{j} \leqslant I_{2}$, respectively. Note that the entangled state $\rho$ by definition does not allow a local description; that is, it cannot be written as statistical mixtures of product states, $\rho \neq \sum_{i} p_{i} \rho_{A}^{i} \otimes \rho_{B}^{i}$. The unfavourable contrast between the state nonlocality and the $A_{i} \otimes B_{j}$ action locality causes the characterization of the state transformation equation (1) to be extremely ambiguous. A different aspect to account for this problem is that the representation of the mixed state in terms of pure states is not unique, making the characterization of the nonlocal parameters (or entanglement monotones [15]) extremly difficult.

Bearing in mind that a proper uniform description for both the states and operators is impossible in terms of local bases, we shall ask instead the following question: is there a profitable way to describe the local operators $A_{i} \otimes B_{j}$ in terms of nonlocal bases so that the concerning problem could become more transparent? This question will be specified clearly in this paper by the underlying language of group representation theory. Note that each local operator $A_{i}\left(B_{j}\right)$ acting on the $N$-dimensional complex space corresponds, up to a real coefficient, to an element of the unimodular linear transformation group $\operatorname{SL}(N, C)$. Thus, the problem stated above can be recast as finding a proper realization, in terms of nonlocal bases, of the homomorphic representation for the direct product group $S L(N, C) \otimes S L(N, C)$.

In this paper we establish such a realization for the group $\operatorname{SL}(2, C) \otimes S L(2, C)$ that completely resolves the entanglement transformation of the binary composite system. We construct, based on the 'spin flip' operation introduced by Wootters [16], a class of pseudoorthonormal bases in the four-dimensional Hilbert space. We then prove that the representative matrices of the group elements of $S L(2, C) \otimes S L(2, C)$ embedded in such pseudo-orthonormal bases are complex orthogonal, and thus establish the homomorphism of $S L(2, C) \otimes S L(2, C)$ onto the group $S O(4, C)$. Finally, we show that the realization of the representation for $S L(2, C) \otimes S L(2, C)$ actually enables the complete characterization for the entanglement transformation of the binary composite systems under LOCC.

Let us start by introducing the following notation. For the states in the space $C^{2} \otimes C^{2}$, define the pseudo inner product as

$$
C\left(\psi_{1}, \psi_{2}\right) \equiv\left\langle\psi_{1} \mid \tilde{\psi}_{2}\right\rangle \text {. }
$$

Here the spin-flipped state $|\tilde{\psi}\rangle$ is introduced according to Wootters' definition [16]

$$
|\tilde{\psi}\rangle=\sigma_{2} \otimes \sigma_{2}\left|\psi^{*}\right\rangle
$$

with $\left|\psi^{*}\right\rangle$ indicating the complex conjugate of $|\psi\rangle$. A state $|\psi\rangle$ is said to be pseudo-normalized if there exists

$$
C(\psi)=\langle\psi \mid \tilde{\psi}\rangle=1
$$

For an arbitrary state $|\phi\rangle$ in $C^{2} \otimes C^{2}$, its pseudo-normalization (denoted as $\|\cdots\|$ hereafter) can be given as $\||\phi\rangle \|=r \mathrm{e}^{\mathrm{i} \theta}|\phi\rangle$, where $r$ is a positive real number and the phase factor $\theta$ is set as $0 \leqslant \theta<\pi$. Note that pseudo-normalization (4) avoids the phase uncertainty which occurs in the common definition of normalization. 
The set of pseudo-orthonormal bases $\left\{\left|x_{i}\right\rangle, i, j=1, \ldots, 4\right\}$ is defined similarly as the conventionally used orthonormal set but with

$$
C\left(x_{i}, x_{j}\right)=\left\langle x_{i} \mid \tilde{x}_{j}\right\rangle=\delta_{i j} .
$$

Obviously, the pseudo-conjugate set $\left\{\left|\tilde{x}_{i}\right\rangle\right\}$ itself constitutes also a set of pseudo-orthonormal bases. The pseudo-orthogonality relation (5) can be alternatively stated as the set of bases that are biorthogonal to their pseudo-conjugate set. From the biorthogonality of the dual bases it suffices to see that the pseudo-orthonormal basis constitutes a complete set in the space $C^{2} \otimes C^{2}$. Actually, given an arbitrary set of linearly independent vectors $\left\{\left|\alpha_{i}\right\rangle, i=1, \ldots, 4\right\}$, one can construct immediately the pseudo-orthonormal basis $\left\{\left|x_{i}\right\rangle, i=1, \ldots, 4\right\}$ via the following pseudo 'Schmidt orthogonalization' procedure by setting that

$$
\begin{aligned}
& \left|x_{1}\right\rangle=\|\left|\alpha_{1}\right\rangle \| \\
& \left|x_{n}\right\rangle=\|\left(I-\sum_{i=1}^{n-1}\left|x_{i}\right\rangle\left\langle\tilde{x}_{i}\right|\right)\left|\alpha_{n}\right\rangle \| \quad n=2,3,4 .
\end{aligned}
$$

Before proceeding, let us mention that the special set of maximally entangled states, the so-called 'magic basis' introduced by Bennett et al [5],

$$
\begin{array}{ll}
\left|e_{1}\right\rangle=\frac{\sqrt{2}}{2}(|00\rangle+|11\rangle) & \left|e_{2}\right\rangle=\mathrm{i} \frac{\sqrt{2}}{2}(|00\rangle-|11\rangle) \\
\left|e_{3}\right\rangle=\mathrm{i} \frac{\sqrt{2}}{2}(|01\rangle+|10\rangle) & \left|e_{4}\right\rangle=\frac{\sqrt{2}}{2}(|01\rangle-|10\rangle)
\end{array}
$$

is just the simplest pseudo-orthonormal basis. In this case the dual bases are completely overlapped: $\left|e_{i}\right\rangle=\left|\tilde{e}_{i}\right\rangle$, hence the pseudo-orthonormal set $\left\{\left|e_{i}\right\rangle\right\}$ recovers the common orthonormal basis. Remarkably, it is shown that this magic basis possesses very curious properties and has been employed to quantify the entanglement of some two-qubit states in earlier studies $[5,17]$. Note that the unimodular unitary transformations acting locally on the two subsystems

$$
U_{A} \otimes U_{B}=\left(\cos \theta_{a} I_{2}+\mathrm{i} \sin \theta_{a} \vec{r}_{a} \cdot \sigma_{a}\right) \otimes\left(\cos \theta_{b} I_{2}+\mathrm{i} \sin \theta_{b} \vec{r}_{b} \cdot \sigma_{b}\right)
$$

correspond to real orthogonal matrices in terms of the above set of bases (7). In the context of group theory, the homomorphism between the groups $S U(2) \otimes S U(2)$ and $S O$ (4) is well known. The magic basis establishes in fact an explicit realization for such a homomorphism, namely, the two-to-one mapping of $S U(2) \otimes S U(2)$ onto $S O(4)$ in the four-dimensional complex space. The kernel of the homomorphism is just the centre of the group $S U(2) \otimes S U(2)$ consisting of the elements $\left\{I_{2} \otimes I_{2},-I_{2} \otimes-I_{2}\right\}$. In this stage, there is no curiosity that any real orthogonal combinations of the magic basis correspond to local unitary transformations on the subsystems and hence yield still maximally entangled states (another magic basis).

Let us now explore the implication of the aforementioned pseudo-orthonormal basis $\left\{\left|x_{j}\right\rangle\right\}$ with equation (5), which is obviously a nontrivial extension of the magic basis. To this end, consider the class of transformations that preserves the pseudo-inner product

$$
C(R x, R y)=C(x, y) .
$$

It is equivalent to saying, by definition (2), that the operators $R$ satisfy

$$
R^{\dagger} \tilde{R}=\tilde{R} R^{\dagger}=I
$$

Theorem 1. The statements below are equivalent:

(i) Operator $R$ preserves the pseudo-inner product;

(ii) Operator $R$ transforms a set of pseudo-orthonormal bases $\left\{\left|x_{i}\right\rangle\right\}$ into another such set $\left\{\left|x_{i}^{\prime}\right\rangle\right\}$ 
(iii) The representation of an operator $R \rightarrow D(R)$ in terms of the biorthogonal dual bases $\left\{\left|x_{i}\right\rangle\right\}$ and $\left\{\left|\tilde{x}_{j}\right\rangle\right\}$, defined as $D_{i j}(R) \equiv\left\langle\tilde{x}_{i}|R| x_{j}\right\rangle$, is a $4 \times 4$ complex orthogonal matrix. Namely, the matrix $D(R)$ satisfies

$$
D(R) D^{T}(R)=D^{T}(R) D(R)=I
$$

where the superscript ' $T$ ' denotes the matrix transpose.

Proof. The equivalence between statements (i) and (ii) is evident, merely regarding that the set of pseudo-orthonormal states constitutes a complete set of bases for the space $C^{2} \otimes C^{2}$. The equivalence between (i) and (iii) is proved as follows. Firstly, given the transformation $R$ preserving the pseudo-inner product, one has the representative matrix

$$
\begin{aligned}
\left(D(R) D^{T}(R)\right)_{i j} & =\sum_{k=1}^{4}\left\langle\tilde{x}_{i}|R| x_{k}\right\rangle\left\langle\tilde{x}_{j}|R| x_{k}\right\rangle \\
& =\sum_{k=1}^{4}\left\langle\tilde{x}_{i}|R| x_{k}\right\rangle\left\langle\tilde{x}_{k}\left|\tilde{R}^{\dagger}\right| x_{j}\right\rangle \\
& =\left\langle\tilde{x}_{i}\left|R \tilde{R}^{\dagger}\right| x_{j}\right\rangle=\delta_{i j}
\end{aligned}
$$

where we have used the fact that $\sum_{k=1}^{4}\left|x_{k}\right\rangle\left\langle\tilde{x}_{k}\right| \equiv I$ and $R \tilde{R}^{\dagger}=\left(\tilde{R} R^{\dagger}\right)^{\dagger}=I$. Reciprocally, from statement (iii) and noting that the operator $R$ can be expressed as $R=\sum_{i j} D_{i j}(R)\left|x_{i}\right\rangle\left\langle\tilde{x}_{j}\right|$ one can obtain immediately that

$$
\begin{aligned}
R^{\dagger} \tilde{R} & =\sum_{i, j, k=1}^{4} D_{i j}(R) D_{i k}(R)\left|\tilde{x}_{j}\right\rangle\left\langle x_{k}\right| \\
& =\sum_{j=1}^{4}\left|\tilde{x}_{j}\right\rangle\left\langle x_{j}\right|=I .
\end{aligned}
$$

We have thus completed the proof.

Theorem 1 is important as it discloses the intrinsic connection between the complex orthogonal matrix and the linear transformation that preserves the pseudo inner product. As one can see, the aggregate of all complex orthogonal matrices constitutes the linear transformation group $S O(4, C)$, which is a direct extension of the real orthogonal group $S O(4)$ in the magic basis. Physically, the presence of a pseudo-orthonormal basis actually renders a homomorphic realization for the direct product group $S L(2, C) \otimes S L(2, C)$ onto $S O(4, C)$.

Theorem 2. The homomorphism between the transformation group $\operatorname{SL}(2, C) \otimes S L(2, C)$ and the complex $S O(4, C)$ can be realized via the pseudo-orthonormal basis.

Proof. Let us start with a readily verified fact that every element of $S L(2, C)$, which is a complex $2 \times 2$ matrix with unit determinant, satisfies the relation

$$
A^{\dagger} \tilde{A}=I_{2} \quad B^{\dagger} \tilde{B}=I_{2} .
$$

Thus the operator of the direct product $A \otimes B$ satisfies equation (10) and, hence, preserves the pseudo-inner product. According to theorem 1, the above arguments amount to the realization, via the pseudo-orthogonal basis, of the correspondence for each element $A \otimes B$ of the group $S L(2, C) \otimes S L(2, C)$ to a unique complex orthogonal matrix $D$.

The proof of the reverse relation that for each matrix $D$ of $S O(4, C)$ there correspondences the element $A \otimes B$ of the group $S L(2, C) \otimes S L(2, C)$ is less straightforward. Note that according 
to theorem 1 the complex orthogonal matrix $D$ induces the transformation $R$ that preserves the pseudo-inner product. Therefore, to prove the existence of $S O(4, C) \rightarrow S L(2, C) \otimes S L(2, C)$ correspondence, it requires only to demonstrate that the operator $R$ can be decomposed into the product form $A \otimes B$. To this end, let us introduce:

Lemma 1. Any operator $H$ that transforms a set of pseudo-orthonormal bases $\left\{\left|x_{i}\right\rangle\right\}$ into its dual set $\left\{\left|\tilde{x}_{i}\right\rangle\right\}$ can be decomposed into a product form. Such an operator $H$ is Hermitian and can be written as $H=\sum_{i=1}^{4}\left|\tilde{x}_{i}\right\rangle\left\langle\tilde{x}_{i}\right|$.

This lemma was proved previously in [18] through directly decomposing the operator into product form. Note the concerning operator $R$ now takes the form $R=\sum_{i=1}^{4}\left|x_{i}^{\prime}\right\rangle\left\langle\tilde{x}_{i}\right|$, which is generally not Hermitian. It would be helpful to introduce the polar decomposition for the operator, $R=\sqrt{R R^{\dagger}} U$, where $U$ is a unitary operator. Then, the required property for $R$ can be proved by showing that both the Hermitian part $\sqrt{R R^{\dagger}}$ and the unitary $U$ can be decomposed into the product forms. For the Hermitian part, we have explicitly that

$$
R R^{\dagger}=\sum_{i, j=1}^{4}\left|x_{i}^{\prime}\right\rangle\left\langle\tilde{x}_{i} \mid \tilde{x}_{j}\right\rangle\left\langle x_{j}^{\prime}\right| .
$$

Suppose the pseudo-orthonormal basis $\left\{\left|\tilde{x}_{i}\right\rangle\right\}$ is related to the magic basis $\left\{\left|e_{i}\right\rangle\right\}$ by a transformation

$$
\left|\tilde{x}_{i}\right\rangle=\sum_{j=1}^{4} S_{i j}\left|e_{j}\right\rangle
$$

where $S^{T} S=I$ by theorem 1 . We can then obtain

$$
\begin{aligned}
R R^{\dagger} & =\sum_{i, j, m, n} S_{i m}^{*} S_{j n}\left|x_{i}^{\prime}\right\rangle\left\langle e_{m} \mid e_{n}\right\rangle\left\langle x_{j}^{\prime}\right| \\
& =\sum_{m}\left(\sum_{i} S_{i m}^{*}\left|x_{i}^{\prime}\right\rangle\right)\left(\sum_{j} S_{j m}\left\langle x_{j}^{\prime}\right|\right) \\
& =\sum_{m=1}^{4}\left|y_{m}\right\rangle\left\langle y_{m}\right| .
\end{aligned}
$$

Here $\left|y_{m}\right\rangle \equiv \sum_{i} S_{i m}^{*}\left|x_{i}^{\prime}\right\rangle$, which obviously constitutes another set of pseudo-orthonormal bases satisfying $\left\langle y_{m} \mid \tilde{y}_{n}\right\rangle=\delta_{m n}$. Consequently, according to lemma 1, the operator $R R^{\dagger}$ (hence its square root $\sqrt{R R^{\dagger}}$ ) can be decomposed into the product form. To show the unitary part $U$ of the operator $R$ is also factorizable, let us write

$$
I=R^{\dagger} \tilde{R}=U^{\dagger}\left(\sqrt{R R^{\dagger}}\right)\left(\widetilde{\sqrt{R R^{\dagger}}}\right) \tilde{U}=U^{\dagger} \tilde{U}
$$

which leads to $U=\tilde{U}$. Therefore, $U$ must transform a magic basis into another set of magic basis. As mentioned earlier, $U$ just belongs to the class of unitary transformations that act locally on the subsystems: $U=U_{A} \otimes U_{B}$. Note that

$$
R^{\dagger} R=U^{\dagger}\left(R R^{\dagger}\right) U
$$

Therefore, once the product form of $R^{\dagger} R$ and $R R^{\dagger}$ is obtained, the distinct forms of $U_{A}$ and $U_{B}$ can be worked out consequently. We have now concluded the required property that the operator $R$ preserving a pseudo-inner product does have a product form $A \otimes B$. 
The above proof depicts also that the transformation of $S O(4, C)$ under the pseudoorthonormal basis does correspond to the physical operation acting on the two subsystems locally. Obviously, the mapping $R=A \otimes B \rightarrow D(R)$ preserves the group multiplication. We have thus completed the proof of theorem 2, the homomorphism of $S L(2, C) \otimes S L(2, C) \sim$ $S O(4, C)$. More specifically, by viewing the unimodular constraint of $\operatorname{det} A=\operatorname{det} B=1$, the mapping between the elements of the group $S L(2, C) \otimes S L(2, C)$ and that of $S O(4, C)$ is two-to-one, reflected by the kernel $\left\{I_{2} \otimes I_{2},-I_{2} \otimes-I_{2}\right\}$.

With the group representation theory established, we are now in the position to investigate the entanglement transformation for the binary composite system. In practice, the general LOCC process (1) can be decomposed into a sequence of elementary actions of generalized measurements with two outcomes. Commonly, as in the filtering process [19], only one outcome will produce the desired result with a finite probability. We thus consider simply the following transformation:

$$
\sigma=\frac{A \otimes B \rho A^{\dagger} \otimes B^{\dagger}}{\operatorname{tr}\left(\rho A^{\dagger} A \otimes B^{\dagger} B\right)} .
$$

Suppose the matrix representation of the state $\rho$ in a set of pseudo-orthonormal bases $\left\{\left|\tilde{x}_{i}\right\rangle\right\}$ is given by $\left\langle\tilde{x}_{i}|\rho| \tilde{x}_{j}\right\rangle=h_{i j}^{\rho}$, or explicitly

$$
\rho=\sum_{i, j=1}^{4} h_{i j}^{\rho}\left|x_{i}\right\rangle\left\langle x_{j}\right| .
$$

Since the operator $A \otimes B$ just transforms the basis $\left\{\left|x_{i}\right\rangle\right\}$ into another pseudo-orthonormal basis, say $\left\{\left|y_{i}\right\rangle\right\}$, one has

$$
\begin{aligned}
\sigma & =N \sum_{i, j=1}^{4} h_{i j}^{\rho}\left|y_{i}\right\rangle\left\langle y_{j}\right| \\
& =N \sum_{i, j=1}^{4}\left(S^{T} h^{\rho} S^{*}\right)_{i j}\left|x_{i}\right\rangle\left\langle x_{j}\right|
\end{aligned}
$$

where $N$ is the trace norm factor and $S^{T} S=I$. Thus, we can now conclude the following fact:

Theorem 3. The state transformation $\rho \rightarrow \sigma$ for the composite binary system can be realized under LOCC iff there exists a complex orthogonal matrix $S$ such that

$$
\frac{h^{\sigma}}{\operatorname{det} h^{\sigma}}=\frac{S h^{\rho} S^{\dagger}}{\operatorname{det} h^{\rho}}
$$

where $h^{\rho}$ and $h^{\sigma}$ are representative matrices of $\rho$ and $\sigma$ in the pseudo-orthonormal basis, respectively.

Moreover, note that the density matrix $\rho$ can always be 'diagonalized' in a certain set of pseudo-orthonormal bases [16]. That is, that

$$
\rho=\sum_{i=1}^{4} \lambda_{i}^{\rho}\left|x_{i}^{\rho}\right\rangle\left\langle x_{i}^{\rho}\right|
$$

where $\lambda_{i}^{\rho}$ in decreasing order have been shown to be related to the volume of a certain measure of entanglement in $\rho$. According to theorem 3 , we have $\operatorname{diag}\left\{\lambda_{1}^{\rho}, \lambda_{2}^{\rho}, \lambda_{3}^{\rho}, \lambda_{4}^{\rho}\right\} \propto$ 
$\operatorname{diag}\left\{\lambda_{1}^{\sigma}, \lambda_{2}^{\sigma}, \lambda_{3}^{\sigma}, \lambda_{4}^{\sigma}\right\}$. Thus it can be concluded concisely that the transformation $\rho \rightarrow \sigma=$ $\sum_{i=1}^{4} \lambda_{i}^{\sigma}\left|x_{i}^{\sigma}\right\rangle\left\langle x_{i}^{\sigma}\right|$ can be achieved by LOCC iff $\lambda_{i}^{\rho} / \lambda_{j}^{\rho}=\lambda_{i}^{\sigma} / \lambda_{j}^{\sigma}$. That the ratios $\lambda_{i} / \lambda_{j}$ are invariant under the LOCC operations was first proved in [11]. The detailed local actions can be acquired straightforwardly as

$$
\frac{A \otimes B}{\sqrt{\operatorname{det} A \times \operatorname{det} B}}=\sum_{i=1}^{4}\left|x_{i}^{\sigma}\right\rangle\left\langle\tilde{x}_{i}^{\rho}\right| .
$$

In summary, this work provides a fruitful application of the group representation theory to the study of the LOCC entanglement transformation. We have shown, for the simplest binary composite system, that the local operators acting on the subsystems are represented by complex orthogonal matrices in terms of the set of bases that are pseudo orthonormalized. This leads explicitly to the notable realization of the homomorphic representation for the direct product group $S L(2, C) \otimes S L(2, C)$ onto the group $S O(4, C)$. Consequently, the entanglement transformation for the binary composite system is completely characterized by virtue of the resulting representation theory. Further studies on the entanglement transformation of more complex composite quantum systems, such as the many-pair binary systems and the multipartite composite systems, should resort to the representation theory of Lie group with high dimensions, which will be a subject of future research.

\section{Acknowledgments}

This work is financially supported by a post-doctoral fellowship from HKUST, the Postdoctoral Science Foundation of China, the special funds for major state basic research project no G001CB3095 of China, and a special grant from Chinese Academy of Sciences to distinguished young researchers.

\section{References}

[1] Ekert A and Jozsa R 1996 Rev. Mod. Phys. 68733

[2] Bennett C H, Brassard G, Crepeau C, Jozsa R, Peres A and Wootters W K 1993 Phys. Rev. Lett. 701895

[3] Steane A 1998 Rep. Prog. Phys. 61117

[4] Bennett C H, Bernstein H J, Popescu S and Schumacher B 1996 Phys. Rev. A 532046

Bennett C H, Brassard G, Popescu S, Schumacher B, Smolin J A and Wootters W K 1996 Phys. Rev. Lett. 76722

[5] Bennett C H, DiVincenzo D P, Smolin J A and Wootters W K 1996 Phys. Rev. A 543824

[6] Horodecki M, Horodecki P and Horodecki R 2000 Phys. Rev. Lett. 842014

[7] Donald M, Horodecki M and Rudolph O 2002 J. Math. Phys. 434252

[8] Nielsen M A 1999 Phys. Rev. Lett. 83436

[9] Vidal G 1999 Phys. Rev. Lett. 831046

[10] Vidal G, Jonathan D and Nielsen M A 2000 Phys. Rev. A 62012304

[11] Linden N, Massar S and Popescu S 1998 Phys. Rev. Lett. 813279

[12] Kent A, Linden N and Massar S 1999 Phys. Rev. Lett. 832656

[13] Verstraete F, Dehaene J and DeMoor B 2001 Phys. Rev. A 61 010101(R)

[14] Schumacher B 1996 Phys. Rev. A 542614

[15] Vidal G 2000 J. Mod. Opt. 47355

[16] Wootters W K 1998 Phys. Rev. Lett. 802245

[17] Hill S and Wootters W K 1997 Phys. Rev. Lett. 785022

[18] Cen L X, Wu N J, Yang F H and An J H 2002 Phys. Rev. A 65052318

[19] Gisin N 1996 Phys. Lett. A 210151 